\title{
Correction and Addendum
}

to the Paper

\section{Unsteady flow of an elastico-viscous liquid}

By N.D. Waters and M.J.King

Rheologica Acta 9, 345 (1970)

Unfortunately the figure legends of this paper have been omitted by the printers. They are now reproduced as follow:

In all figures the discontinuous lines represent the Newtonian solutions.

Fig. 4. Generation of flow due to a tangential force. $S_{1}=0.08, S_{2}=0.02$, (a) $t_{1}=0.1$ (b) $t_{1}=0.2$ (c) $t_{1}=$ $0.3(\mathrm{~d}) t_{1}=0.5(\mathrm{e}) t_{1}=\infty$.

Fig. 5. $S_{1}=2.0, S_{2}=0.5$, (a) $t_{1}=0.1$ (b) $t_{1}=0.2$ (c) $t_{1}=0.4$ (d) $t_{1}=1.5$ (e) $t_{1}=2.5$ (f) $t_{1}=\infty$,

…- are the Newtonian solutions for $t_{1}=0.1$ and 1.5.

Fig. 6. The velocity at $y_{1}=1$ against time when $S_{1}=2.0$ and (a) $S_{2}=0.3(\mathrm{~b}) S_{2}=1.0$ (c) $S_{2}=1.6$.

Fig. 7. Decay of the steady flow due to a tangential force. $S_{1}=0.08, S_{2}=0.02$, (a) $t_{1}=0.1$ (b) $t_{1}=0.2$ (c) $t_{1}=0.4$ (d) $t_{1}=0.6$ (e) $t_{1}=\infty$.

Fig. 8. $S_{1}=2.0, \quad S_{2}=0.5$, (a) $t_{1}=0.1$ (b) $t_{1}=0.2$ (c) $t_{1}=0.5$ (d) $t_{1}=1.5$ (e) $t_{1}=2.4$,

..... are the New tonian solutions for $t_{1}=0.1$ and $t_{1}=1.5$.
Fig. 9. Velocity at $y_{1}=1$ against time when $S_{1}=2.0$ and (a) $S_{2}=0.3$ (b) $S_{2}=1.0$ (c) $S_{2}=1.6$.

Fig. 10. Generation of flow in a channel. $S_{1}=0.04$, $S_{2}=0.02$, (a) $t_{1}=0.06$ (b) $t_{1}=0.1$ (c) $t_{1}=0.2$ (d) $t_{1}=\infty$.

Fig. 11. $S_{1}=0.4, S_{2}=0.1$, (a) $t_{1}=0.1$ (b) $t_{1}=0.4$ (c) $t_{1}=0.6$ (d) $t_{1}=0.7$ (e) $t_{1}=\infty$, -.... is the Newtonian solution for $t_{1}=0.1$.

Fig. 12. Velocity at $y_{1}=0.5$ wher $S_{2}=0.3$ and (a) $S_{2}=0.04$ (b) $S_{2}=0.1$ (c) $S_{2}=0.2$.

Fig. 13. Decay of steady flow in a channel. $S_{1}=0.04$, $S_{2}=0.02$, (a) $t_{1}=0.05$ (b) $t_{1}=0.1$ (c) $t_{1}=0.2$ (d) $t_{1}=\infty$.

Fig. 14. $S_{1}=0.4, S_{2}=0.1$ (a) $t_{1}=0.07$ (b) $t_{1}=0.12$ (c) $t_{1}=0.4$ (d) $t_{1}=0.6$ (e) $t_{1}=\infty$,

...... is the Newtonian solution for $t_{1}=0.07$.

Fig. 15. Velocity at $y_{1}=0.5$ when $S_{1}=0.3$ and (a) $S_{2}=0.04$ (b) $S_{2}=0.1$ (c) $S_{2}=0.2$.

\section{Preliminary announcement}

\author{
The autumn conference 1971 of The British Society of Rheology \\ presumably will be held on September 20-23, 1971.
}

The main theme of the conference will be the general rheology with a special emphasis on the rheology of polymers and dispersed systems as well as the rheometry.

Papers to be read should be announced together with the sending of the abstracts until May 1, 1971 to

Mr. A. PLEASE, Materials Section, Road Research Laboratory Crowthorne, Berks., RG11 6AU (England) 